\title{
GEOMETRICAL FRAMEWORK OF QUANTIZATION PROBLEM
}

\author{
M. Grigorescu
}

The basic elements of the geometric approach to a consistent quantization formalism are summarized, with reference to the methods of the old quantum mechanics and the induced representations theory of Lie groups. A possible relationship between quantization and phase-space discretization is briefly discussed.

PACS: 04.60.Ds, 02.40.-k, 02.20.Qs 


\section{Introduction}

The notion of quantization has appeared at the beginning of the last century in the theory of thermal radiation, when M. Planck has formulated the hypothesis of energy quanta ${ }^{1}: \epsilon=\mathrm{h} \nu, \mathrm{h}=6.626 \times 10^{-34} \mathrm{~J} \cdot \mathrm{s}[21]$. The existence of $\mathrm{h}$ was considered in statistical mechanics as evidence for a granular structure of the $2 n$-dimensional phase-space, composed of elementary cells ("quantum states") of volume $\mathrm{h}^{n}$. For integrable systems with multiple periodic motions, such as the hydrogen atom, this structure was provided by the quantization rules of the old quantum mechanics. Relativistic effects have also been included, as a correction to Balmer's formula, due to the variation of mass with velocity, was introduced by Bohr [3], and the relativistic Kepler problem was quantized by Sommerfeld, applying integrality constraints to the action invariants [25].

In the algebraic (Dirac) approach to quantum mechanics, the observables are represented by elements of the set $\mathcal{F}(M)$ of smooth real functions over the classical (momentum) phase-space $(M, \omega), M=T^{*} Q,\left(Q=\mathbb{R}^{3}\right)$, with $\omega$ the globally defined symplectic form. Let $X_{f}$ be the vector field determined by $i_{X_{f}} \omega=\mathrm{d} f$, and $\mathrm{L}_{X}$ the Lie derivative with respect to $X$. As $\mathcal{F}(M)$ becomes a Lie algebra with respect to the Poisson bracket $\{*, *\}$, $\{f, g\}=\left\langle d f, X_{g}\right\rangle=\omega\left(X_{f}, X_{g}\right)=-\mathrm{L}_{X_{f}} g, \quad f, g \in \mathcal{F}(M)$, the full quantization of $M$ was defined as a $\mathbb{R}$-linear map $f \mapsto \hat{f}$ from $\mathcal{F}(M)$ to a set $\mathcal{A}(\mathcal{H})$ of symmetric operators on the Hilbert space $\mathcal{H}$, having the following properties [1]:

1. the map ^ $: \mathcal{F}(M) \mapsto \mathcal{A}(\mathcal{H})$ is injective.

2. $[\hat{f}, \hat{g}]=\mathrm{i} \hbar\{f, g\} ; f, g \in \mathcal{F}(M)$.

3. $\hat{1}=I$, for $f=1$, constant on $M$, and $I$ the identity operator on $\mathcal{H}$.

4. $\hat{q}_{k}, \hat{p}_{k}, k=1,2,3$ act irreducibly on $\mathcal{H}$.

It is presumed that once ${ }^{\wedge}$ and $\mathcal{H}$ are found, the quantum dynamics with respect to the classical time $[6,4]$ is given by the Schrödinger equation, and the scalar product in $\mathcal{H}$ has the statistical interpretation of probability amplitude.

\footnotetext{
${ }^{1}$ It is important to remark that unlike the Nyquist formula for a linear electric circuit (RLC), the Planck distribution (accurately retrieved in the $2.7 \mathrm{~K}$ cosmic microwave background spectrum) contains no zero-point energy term, and the photon energy $\epsilon=\mathrm{h} \nu=\mathrm{h} c / \lambda$ corresponds to a free relativistic particle with momentum $|\mathbf{p}|=\mathrm{h} / \lambda$ and Hamiltonian $c|\mathbf{p}|$ (arXiv0912.1998). Also, for thermal radiation the wavelength $\lambda$ is the significant variable, as the Wien displacement law $\lambda_{\max } T=\mathrm{hc} / 4.965 k_{B}=2.9 \mathrm{~mm} \cdot \mathrm{K}$ describes the maximum of the spectral density (in vacuum) expressed as a function of $\lambda$.
} 
In classical nonrelativistic statistical mechanics, a Brownian particle can be described by a time-dependent distribution function $f \geq 0$ defined on the phase-space $M=T^{*} \mathbb{R}^{3}$, evolving according to the Fokker-Planck equation. Though, at zero temperature both classical and quantum distributions arise as two different types of "functional coherent states" $f_{0}, f_{\psi}$ for the classical Liouville equation [8]. These are solutions associated with "action waves"

$\mathrm{n}^{[S]}$, respectively "quantum waves" $\psi=\sqrt{\mathrm{n}} \exp (\mathrm{i} S / \hbar)$, expressed in terms of only two functions of coordinates and time: the localization probability density in the coordinate space $\mathrm{n}(\mathbf{q}, t)$, and the local "momentum potential" $S(\mathbf{q}, t)$. Moreover, these classes are related, as the action distributions turn into quantum distributions (Wigner functions) when the configuration space $Q=\mathbb{R}^{3}$ is discretized. However, by contrast to the action distributions, the Wigner distributions $\mathrm{f}_{\psi}$ remain the same as functionals of $\psi$ during time evolution only for polynomial potentials of degree at most 2 . This limitation also appears in the canonical quantization, as the van Hove theorem $[1,10]$ states the incompatibility between the conditions $1,2,3,4$. Thus, it is possible to fulfill the first three conditions, obtaining a "prequantization", but then the Heisenberg algebra $\mathfrak{H} \equiv\{q, p, 1\}$ is represented with infinite multiplicity. Also, if only the last three conditions are retained, then the map ^ should be restricted to some subalgebra $\mathcal{F}_{P} \subset \mathcal{F}(M)$, containing $\mathfrak{H}$.

This work ${ }^{2}$ presents, following [14] as main reference, the geometrical framework in which the "action" and the "quantum" phase-space distributions are defined. The main concepts applied to the prequantization of Hamiltonian dynamical systems are recalled in Section 2. The reduction of the prequantum Hilbert space $\mathcal{H} \simeq \mathrm{L}^{2}\left(M, \omega^{n}\right)$, to the quantum Hilbert space $\mathcal{H}_{P} \simeq \mathrm{L}^{2}(Q)$, is considered in Section 3. The transition from classical to quantum distributions by phase-space discretization is outlined in Section 4. Conclusions are summarized in Section 5.

\section{The Prequantization}

\subsection{Equivalence Classes of Line Bundles}

Let $M$ be a $C^{\infty}$ differentiable manifold, separable and connected. A line bundle on $M$ is a vector bundle

\footnotetext{
${ }^{2}$ The next two sections are based on the notes of the seminar "Classical limit and quantization methods" given in 1989 at the Institute of Atomic Physics from Bucharest. The introductory section of this seminar, not included here, can be found in [6].
} 


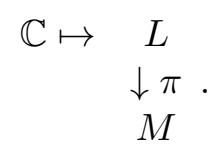

The projection map $\pi$ is smooth, and for any $p \in M, L_{p}=\pi^{-1}(p)$ (the fiber in $p$ ) is a one-dimensional vector space over $\mathbb{C}$.

On $L$, as manifold, we can introduce local coordinates. Let $\mathcal{U}=\left\{U_{i}, i \in\right.$ $I\}$, be an open covering of $M$, and $s_{i}: U_{i} \mapsto L$ smooth non-vanishing sections, such that the map $\sigma_{i}: \mathbb{C} \times U_{i} \mapsto \pi^{-1}\left(U_{i}\right), \sigma_{i}(z, p)=z s_{i}(p)$, is a diffeomorphism. The set of pairs $\left\{\left(U_{i}, s_{i}\right), i \in I\right\}$ defines a local system for the bundle $L$.

Let $\Gamma_{L}$ be the space of smooth sections $s: M \mapsto L$. For the local system $\left(U_{i}, s_{i}\right)$ any $s \in \Gamma_{L}$ can be written on $U_{i}$ as $s=\psi_{i} s_{i}$, where $\psi_{i} \in \mathcal{F}_{c}\left(U_{i}\right)$ is a complex function on $U_{i}$. The collection $\left\{\psi_{i}\right\}_{i \in I}$ represents the local coordinates of $s$.

On $U_{i} \cap U_{j}$ the local system defines by the relation $s_{i}=c_{i j} s_{j}$ the transition functions $c_{i j} \in \mathcal{F}_{c}^{*}\left(U_{i} \cap U_{j}\right)$. These functions should satisfy the relationships

$$
c_{i j}=c_{j i}^{-1}, \quad c_{i j} c_{j k}=c_{i k}
$$

on $U_{i} \cap U_{j}$, respectively on $U_{i} \cap U_{j} \cap U_{k}$. If expressed in the form $c_{i j}=$ $\exp \left(\mathrm{i} q_{i j} / \hbar\right),(\hbar=\mathrm{h} / 2 \pi=1 / 2 \pi$, as we take $\mathrm{h}=1)$, we can see that the new functions $q_{i j}$ provide a constant with integer values, denoted $a_{i j k}$,

$$
a_{i j k}=q_{i j}+q_{j k}-q_{i k} \in \mathbb{Z}
$$

on any intersection $U_{i} \cap U_{j} \cap U_{k} \neq \emptyset$.

Two line bundles $L^{1}$ and $L^{2}$ on $M$ are equivalent if there exists a diffeomorphism $\tau: L^{1} \mapsto L^{2}$ such that for any $p \in M$, the map $\tau$ induces a linear isomorphism $L_{p}^{1} \mapsto L_{p}^{2}$. The set of equivalence classes of line bundles on $M$ is denoted $\mathcal{L}(M)$.

If $c_{i j}^{1}, c_{i j}^{2}$ are the transition functions for $L^{1}$, respectively $L^{2}$, then the two are equivalent iff there exists $\lambda_{i}=s_{i}^{2} / s_{i}^{1}, \lambda_{i} \in \mathcal{F}_{c}^{*}\left(U_{i}\right)$ (the set of nonvanishing complex functions on $U_{i}$ ), such that $c_{i j}^{2}=\lambda_{i} c_{i j}^{1} \lambda_{j}^{-1}$. Using this result it can be proved [14] that there exists a one-to-one mapping $\kappa: \mathcal{L}(M) \mapsto H^{2}(M, \mathbb{Z})$, which assigns to any element $\ell=[L] \in \mathcal{L}(M)$ the Cech cohomology class $[a] \in H^{2}(M, \mathbb{Z})[12]$ of the function $a_{i j k}$ associated with $L$. In particular, $L$ is called trivial if equivalent to $\mathbb{C} \times M\left(\Gamma_{L}\right.$ contains a nonvanishing global section). 


\subsection{Line Bundles with Connection}

Let $\chi_{c}(M)$ be the Lie algebra of complex fields on $M$, and $L$ a line bundle on $M$. A connection in the line bundle $\pi: L \mapsto M$ is a linear map $\nabla$ : $\chi_{c}(M) \mapsto \operatorname{End}\left(\Gamma_{L}\right)$ such that

$$
\begin{gathered}
\nabla_{\Phi \xi}=\Phi \nabla_{\xi} \\
\nabla_{\xi}(\Phi s)=\left(\mathrm{L}_{\xi} \Phi\right) s+\Phi \nabla_{\xi} s, \quad \Phi \in \mathcal{F}_{c}(M), \quad s \in \Gamma_{L} .
\end{gathered}
$$

If $\left\{\left(U_{i}, s_{i}\right), i \in I\right\}$ is a local system for $L$, then $\nabla$ is completely specified by its action on the sections $\left\{s_{i}\right\}_{i \in I}$,

$$
\nabla_{\xi} s_{i}=2 \pi \mathrm{i} \alpha_{i}(\xi) s_{i}, \quad \xi \in \chi_{c}(M), \quad i \in I .
$$

The condition (3) implies $\alpha_{i}(\Phi \xi)=\Phi \alpha_{i}(\xi)$, such that the collection of functions $\left\{\alpha_{i}(\xi), i \in I, \xi \in \chi_{c}(M)\right\}$ defines a family of complex 1-forms $\left\{\alpha_{i}\right\}_{i \in I}$, $i_{\xi} \alpha_{i} \equiv\left\langle\alpha_{i}, \xi\right\rangle=\alpha_{i}(\xi)$, associated to the connection $\nabla$. On $U_{i} \cap U_{j}$ we get

$$
\alpha_{i}=\alpha_{j}+\mathrm{d} q_{i j}
$$

and conversly, any family of 1-forms having this property specifies uniquely a connection $\nabla$. Such a family arises by the pull-back of an unique $\mathbb{C}^{*}$ invariant 1-form $\alpha \in \Omega^{1}\left(L^{*}\right)$, called connection form. Here $\Omega^{k}\left(L^{*}\right)$ denotes the set of $k$-forms on the manifold $L^{*}=\left\{\cup_{p \in M} L_{p}^{*} ; L_{p}^{*}=L_{p}-\{0\}\right\}$. The form $\alpha$ is globally defined on $L^{*}$, and $s_{i}^{*} \alpha=\alpha_{i}, i \in I$.

If $\left(L^{1}, \alpha^{1}\right),\left(L^{2}, \alpha^{2}\right)$, are line bundles on $M$ with connection forms $\alpha^{1}$, $\alpha^{2}$, then there exists a diffeomorphism $\tau: L^{1} \mapsto L^{2}$ such that $\tau$ induces a linear isomorphism

$$
L_{p}^{1} \mapsto L_{\pi\left(\tau\left(L_{p}^{1}\right)\right)}^{2}
$$

and $\tau^{*} \alpha^{2}=\alpha^{1}$.

One should note that any equivalence $(L, \alpha) \mapsto\left(L, \alpha^{1}\right)$ is specified by a function $\Phi \in \mathcal{F}_{c}^{*}(M)$, such that

$$
\tau_{\Phi}^{*} \alpha^{1}=\alpha^{1}-\frac{1}{2 \pi \mathrm{i}} \frac{\mathrm{d} \tilde{\Phi}}{\tilde{\Phi}}=\alpha, \quad \tilde{\Phi}=\pi^{*}(\Phi)
$$

and $\tau_{\Phi}(x)=\Phi(\pi x) x, x \in L$. In particular, $\tau_{\Phi}:(L, \alpha) \mapsto(L, \alpha)$ is an equivalence iff $\Phi$ is a complex constant on $M$.

The family of 1-forms $\left\{\alpha_{i}\right\}_{i \in I}$, associated to the connection $\nabla$ determines an unique complex 2 -form $\omega$ on $M$, such that

$$
\mathrm{d} \omega=0,\left.\quad \omega\right|_{U_{i}}=\mathrm{d} \alpha_{i}, \quad \pi^{*} \omega=\mathrm{d} \alpha .
$$


Because

$$
\left[\nabla_{\xi}, \nabla_{\eta}\right]-\nabla_{[\xi, \eta]}=2 \pi \mathrm{i} \omega(\xi, \eta), \quad \xi, \eta \in \chi_{c}(M)
$$

$\omega$ is called the curvature form of the connection $\nabla$. If real and nondegenerate, $\omega$ provides a symplectic structure on $M$.

\subsection{Line Bundles with Connection and Hermitian Structure}

A Hermitian structure on $L$ is a function $(*, *): L \times L \mapsto \mathbb{C}$ having the properties:

i) $(*, *)$ induces a structure of 1-dimensional Hilbert space on $L_{p}$, for all $p \in M$.

ii) $|*|^{2}$ is a positive function on $L^{*},|x|^{2} \equiv(x, x), x \in L^{*}$.

Let $\gamma$ be a smooth curve on $M$. The covariant derivative of the section $s \in \Gamma_{L}$ along $\gamma$ is defined by

$$
\frac{D s}{D t}=\nabla_{\dot{\gamma}(t)} s .
$$

For any smooth curve $\gamma$ on $M,\left\{\gamma_{t}, t \in(a, b)\right\}$, the covariant derivative defines a linear isomorphism $\tau_{t^{\prime}, t}: L_{\gamma_{t}} \mapsto L_{\gamma_{t^{\prime}}}$, called parallel transport by

$$
\left.\frac{D s}{D t}\right|_{\gamma_{t}}=\left.\frac{\mathrm{d}}{\mathrm{d} t^{\prime}} \tau_{t, t^{\prime}} s\left(\gamma_{t^{\prime}}\right)\right|_{t^{\prime}=t} .
$$

A section $r=\psi s_{i}$ is autoparallel along $\gamma$ if $\nabla_{\dot{\gamma}} r=0$, or

$$
\psi\left(\gamma_{t}\right)=\mathrm{e}^{-2 \pi \mathrm{i} \int_{0}^{t}\left\langle\alpha_{i}, \dot{\gamma}_{t}\right\rangle} \psi\left(\gamma_{0}\right) .
$$

If $\gamma=\partial \Sigma \subset M$ is closed, contractible on $\Sigma$ to a point, then

$$
\mathcal{Q}_{\gamma}=\mathrm{e}^{-2 \pi \mathrm{i} \int_{\Sigma} \omega}
$$

is the scalar function of parallel transport. Applications to autoparallel sections for the constrained quantum dynamics are presented in [7].

The Hermitian form $(*, *)$ is called $\nabla$-invariant if the parallel transport leaves invariant the inner product on fiber,

$$
\left.\frac{\mathrm{d}}{\mathrm{d} t}\left(\tau_{t, t^{\prime}} s_{\left(\gamma_{t^{\prime}}\right)}^{1}, \tau_{t, t^{\prime}} s_{\left(\gamma_{t^{\prime}}\right)}^{2}\right)\right|_{t^{\prime}=t}=0
$$

or

$$
\mathrm{L}_{\xi}\left(s^{1}, s^{2}\right)=\left(\nabla_{\xi} s^{1}, s^{2}\right)+\left(s^{1}, \nabla_{\xi} s^{2}\right) .
$$


When $s^{1}=s^{2}=s_{i}$ this reduces to

$$
\mathrm{d} \ln \left|s_{i}\right|^{2}=2 \pi \mathrm{i}\left(\alpha_{i}-\bar{\alpha}_{i}\right)
$$

where $\bar{\alpha}_{i}$ is the complex conjugate of $\alpha_{i}$. Thus, $\alpha_{i}-\bar{\alpha}_{i}$ is a real 1 -form, exact on $U_{i}$, the curvature form

$$
\left.\omega\right|_{U_{i}}=\mathrm{d} \alpha_{i}=\mathrm{d} \bar{\alpha}_{i}
$$

is real, and $\mathcal{Q}_{\gamma} \in \mathrm{U}(1)$. Let $[\omega]_{d R} \in H_{d R}^{2}(M, \mathbb{R})$ be the de Rahm cohomology class of $\omega$. In general, the isomorphism between $H_{d R}^{2}(M, \mathbb{R})$ and $H^{2}(M, \mathbb{R})$ associates to a real, closed two-form $\omega$ on $M$, expressed locally as

$$
\left.\omega\right|_{U_{i}}=\mathrm{d} \alpha_{i}, \quad \alpha_{i}=\alpha_{j}+\mathrm{d} f_{i j}, \quad f_{i j}: M \mapsto \mathbb{R}
$$

the class $[\omega] \equiv\left[a^{\omega}\right] \in H^{2}(M, \mathbb{R})$, where $a_{i j k}^{\omega}=f_{i j}+f_{j k}-f_{i k}$ is a real constant on $U_{i} \cap U_{j} \cap U_{k}$. However, if $\omega$ is the curvature of a connection $\nabla$ on a line bundle $L$ with $\nabla$-invariant Hermitian structure, then $a_{i j k}^{\omega}$ is an integer ${ }^{3}$, and $\omega$ specifies an integral cohomology class in $H^{2}(M, \mathbb{R})$.

Conversly, the problem is to what extent a closed, real 2-form $\omega$, satisfying an integrality condition, determines a Hermitian line bundle with connection on $M$. If $\omega$ is integral, then in general $a_{i j k}^{\omega}$ are not integers, but we can find real constants $x_{i j}=-x_{j i}$ on $U_{i} \cap U_{j} \neq \emptyset$, such that

$$
z_{i j k}=a_{i j k}+x_{i j}+x_{j k}-x_{i k}
$$

are integers on $U_{i} \cap U_{j} \cap U_{k} \neq \emptyset$. This result allows one to define a line bundle $L$ on $M$ with the transition functions

$$
c_{i j}=\exp \left(2 \pi \mathrm{i} q_{i j}\right), \quad q_{i j}=f_{i j}+x_{i j}
$$

on $U_{i} \cap U_{j} \neq \emptyset$. Because

$$
\alpha_{i}=\alpha_{j}+\mathrm{d} f_{i j}=\alpha_{j}+\mathrm{d} q_{i j}=\alpha_{j}+\frac{1}{2 \pi \mathrm{i}} \frac{\mathrm{d} c_{i j}}{c_{i j}}
$$

with $\alpha_{i}, \alpha_{j}$ real, there exists on $L$ a connection $\nabla$ defined by the family of 1-forms $\left\{\alpha_{i}\right\}_{i \in I}$, and a $\nabla$-invariant Hermitian structure.

\footnotetext{
${ }^{3}$ If $\omega_{f}$ and $\omega_{f}^{*}$ are $\mathrm{SO}(3,1)$-invariant, dual (electromagnetic) 2-forms on $\mathbb{R}^{3,1}$, then $\left[\omega_{f}\right]_{d R}=0$ (the first group of Maxwell equations) and $\left[\omega_{f}^{*}\right]_{d R} / e$ is integral (electric charge quantization, arXiv0912.1998).
} 
In this formulation, the 1 -forms $\alpha_{i}$ are defined by $\omega$ up to a total differential $\mathrm{d} \Phi_{i}$. If $\alpha_{i}^{\prime}=\alpha_{i}+\mathrm{d} \Phi_{i}$, then $f_{i j}^{\prime}=f_{i j}+\Phi_{i}-\Phi_{j}$, and

$$
c_{i j}^{\prime}=\lambda_{i} c_{i j} \lambda_{j}^{-1}, \quad \lambda_{i}=\mathrm{e}^{2 \pi \mathrm{i} \Phi_{i}}
$$

define a Hermitian line bundle with connection $\left(L^{\prime}, \nabla^{\prime}\right)$, equivalent to $(L, \nabla)$. In specifying this equivalence class there is still an arbitrary due to the way of choosing the constants $x_{i j}$. Thus, the integrality condition allows one to replace $x_{i j}$ by new real constants $x_{i j}^{\prime}=x_{i j}+y_{i j}$, where $y_{i j}+y_{j k}+y_{k i} \in \mathbb{Z}$, and $y_{i j}=-y_{j i}$. The line bundle $L^{\prime}$, specified by the transition functions

$$
c_{i j}^{\prime}=\exp 2 \pi \mathrm{i}\left(f_{i j}+x_{i j}^{\prime}\right)=\mathrm{e}^{2 \pi \mathrm{i} y_{i j}} c_{i j}
$$

is equivalent to $L$ only if $y_{i j}$ has the form $y_{i j}=c_{i}-c_{j}$. Because $y_{i j}+y_{j k}+$ $y_{k i} \neq 0, y_{i j}$ does not specify a cocycle in $C^{1}(M, \mathbb{R})$, but in the exponential it determines a cocycle in $C^{1}(M, \mathrm{U}(1))$. The bundles $L^{\prime}$ and $L$ are equivalent only if this cocycle is coboundary, such that the set of equivalence classes of the Hermitian line bundles whose connection has the same curvature form $\omega$ is parameterized by $H^{1}(M, \mathrm{U}(1))$. This set of equivalence classes is denoted by $\mathcal{L}_{c}(M, \omega)$, and the result presented above states the isomorphism $\mathcal{L}_{c}(M, \omega) \simeq H^{1}(M, \mathrm{U}(1)) \simeq H^{2}(M, \mathbb{Z})$.

Let $\epsilon: H^{2}(M, \mathbb{Z}) \mapsto H^{2}(M, \mathbb{R})$ be the homomorphism induced by the injection $\epsilon: \mathbb{Z} \mapsto \mathbb{R}, \kappa: \mathcal{L}(M) \mapsto H^{2}(M, \mathbb{Z})$ the bijection introduced in subsection 2.1, and $\sigma: \mathcal{L}_{c} \mapsto \mathcal{L}$ the mapping given by $\sigma[(L, \alpha)]=[L]$. In this case, the Weil integrality condition states that if $\omega$ is any real, closed 2 -form on $M$, then:

i) $\mathcal{L}_{c}(M, \omega) \neq\{\emptyset\}$ iff $[\omega] \in H^{2}(M, \mathbb{R})$ is integral.

ii) $\sigma \mathcal{L}_{c}(M, \omega)=\{[L] \in \mathcal{L} ; \epsilon \kappa[L]=[\omega]\}$.

Applications to the calculus of the energy levels for the multidimensionl Kepler problem are presented in $[16,17,18]$.

\subsection{The BWS Condition}

Let $(N, \omega)$ be a reducible presymplectic manifold, and $\left(M^{\prime}, \omega^{\prime}\right)$, with $M^{\prime}=$ $N / K$, the reduced space. Here $K$ is a smooth distribution on $N$, with the tangent space

$$
T_{m} K=\left\{x \in T_{m} N ; i_{x} \omega_{m}=0\right\} .
$$

Proposition 1. A sufficient condition to obtain a quantizable reduction $\left(M^{\prime}, \omega^{\prime}\right)$ of $(N, \omega)$ is

$$
\oint_{\gamma} \theta \in \mathbb{Z}
$$


where $\theta$ is a global 1 -form such that $\omega=\mathrm{d} \theta$, and $\gamma$ is any closed curve contained in a leaf of $K$. If $N$ is simply connected, then (12) is also necessary [30].

For the proof we take a contractible covering $\mathcal{U}=\left\{U_{i}, i \in I\right\}$ of $M^{\prime}$, such that for any $i \in I$ there exists a section $\Sigma_{i}$ in $K$ over $U_{i}$ and a diffeomorphism $\rho_{i}: U_{i} \mapsto \Sigma_{i}$. If $m_{1}, m_{2} \in U_{i} \cap U_{j}$ are two points joined by the curve $c$, then $\rho_{i}(c)$ is a curve in $\Sigma_{i}$, and $\rho_{j}(c)$ is a curve in $\Sigma_{j}$. Moreover, $\rho_{i}\left(m_{1}\right)$ and $\rho_{j}\left(m_{1}\right)$ can be joined by a curve $\gamma_{1}$ in the leaf of $K$ through $m_{1}$, respectively $\rho_{i}\left(m_{2}\right)$ and $\rho_{j}\left(m_{2}\right)$ can be joined by a curve $\gamma_{2}$ in the leaf of $K$ through $m_{2}$. Let $S$ be the surface bounded by $\rho_{i}(c), \rho_{j}(c), \gamma_{1}, \gamma_{2}$, so that $\pi(S)=c$. Because $S \in \operatorname{ker}(\omega)$,

$$
\begin{gathered}
\int_{S} \omega=0=\oint_{\partial S} \theta=\int_{\rho_{i}\left(m_{1}\right)}^{\rho_{i}\left(m_{2}\right)} \theta-\int_{\rho_{j}\left(m_{1}\right)}^{\rho_{j}\left(m_{2}\right)} \theta \\
+\int_{\rho_{i}\left(m_{2}\right)}^{\rho_{j}\left(m_{2}\right)} \theta-\int_{\rho_{i}\left(m_{1}\right)}^{\rho_{j}\left(m_{1}\right)} \theta=f_{j i}\left(m_{2}\right)-f_{j i}\left(m_{1}\right)+\int_{m_{1}}^{m_{2}}\left(\rho_{i}^{*} \theta-\rho_{j}^{*} \theta\right)
\end{gathered}
$$

which yields

$$
\rho_{i}^{*} \theta-\rho_{j}^{*} \theta \equiv \theta_{i}-\theta_{j}=\mathrm{d} f_{i j} .
$$

The 1 -forms $\theta_{i}=\rho_{i}^{*} \theta, \theta_{j}=\rho_{j}^{*} \theta$ on $U_{i} \cap U_{j} \neq\{\emptyset\}$ are related to the symplectic form $\omega^{\prime}=\mathrm{d} \theta_{i}=\mathrm{d} \theta_{j}$. The functions $f_{i j}=-f_{j i}$ on $U_{i} \cap U_{j}$ can be defined by integration along an arbitrary curve contained in the leaf of $K$ over $m$,

$$
f_{i j}(m)=\int_{\rho_{i}(m)}^{\rho_{j}(m)} \theta .
$$

Thus, $f_{i j}+f_{j k}+f_{i k} \in \mathbb{Z}$ as an integral (12) of the 1-form $\theta$ along a closed curve in the leaf of $K$ through $m$, proving that the class $\left[\omega^{\prime}\right] \in H^{2}\left(M^{\prime}, \mathbb{R}\right)$ is integral.

When $N=h^{-1}(E) \subset M$ is the constant energy surface of a classical system on $(M, \omega)$ with Hamiltonian $h$, then (12) is similar to the BohrWilson-Sommerfeld (BWS) condition from the old quantum mechanics.

\subsection{The Prequantum Hilbert Space and Operators Related to Observables}

Let $(M, \omega)$ be a quantizable classical phase-space, in the sense that $[\omega] \in$ $H^{2}(M, \mathbb{R})$ is integral. In this case, on $M$ we can define a Hermitian line 
bundle with connection $(L, \alpha)$. The natural volume element on $M$ is $\epsilon_{\omega}=$ $\omega^{n}$, and for $\omega=\sum_{k=1}^{n} \mathrm{~d} q_{k} \wedge \mathrm{d} p_{k}$,

$$
\epsilon_{\omega}=\mathrm{d} q_{1} \wedge \ldots \wedge \mathrm{d} q_{n} \wedge \mathrm{d} p_{1} \wedge \ldots \wedge \mathrm{d} p_{n} .
$$

If $(*, *)$ denotes the $\nabla$-invariant Hermitian form on $L$, the prequantum Hilbert space $\mathcal{H}$ is defined as the space of all sections $s \in \Gamma_{L}(M)$ for which

$$
\int_{M} \epsilon_{\omega}(s, s)
$$

exists and is finite. The inner product in $\mathcal{H}$ is

$$
<s_{1}, s_{2}>\equiv \int_{M} \epsilon_{\omega}\left(s_{1}, s_{2}\right), \quad s_{1}, s_{2} \in \mathcal{H} .
$$

Let $\mathfrak{e}(L)$ be the Lie algebra of the $\mathbb{C}^{*}$-invariant, real fields on $L^{*}$. By the existence of the connection form $\alpha$ and the projection $\pi_{*}: T L \mapsto T M$, there exists also a linear isomorphism

$$
\mathfrak{e}(L) \mapsto \mathcal{F}_{c}(M) \times \chi(M)
$$

which associates to $\eta \in \mathfrak{e}(L)$ a function $\Phi \in \mathcal{F}_{c}(M)$ and a vector $\xi \in \chi(M)$ such that

$$
\pi^{*} \Phi=-\langle\alpha, \eta\rangle, \quad \xi=\pi_{*} \eta .
$$

Conversly, any function $\Phi \in \mathcal{F}_{c}(M)$ specifies an unique field $\eta_{\Phi} \in \mathfrak{e}(L)$, $\eta_{\Phi} \in \operatorname{ker}\left(\pi_{*}\right)$ by the relation

$$
\left\langle\alpha, \eta_{\Phi}\right\rangle=-\pi^{*} \Phi
$$

and for any $\xi \in \chi(M)$ there exists an unique field $\hat{\xi} \in \mathfrak{e}(L), \hat{\xi}_{x} \in \operatorname{ker}\left(\alpha_{x}\right)$, $x \in L^{*}[14]$

$$
\pi_{*} \hat{\xi}=\xi
$$

Proposition 2. $\mathfrak{e}(L)$ is parameterized by $\mathcal{F}_{c}(M) \times \chi(M)$, such that for any $\Phi \in \mathcal{F}_{c}(M)$ and $\xi \in \chi(M)$,

$$
\begin{aligned}
\eta_{(\Phi, \xi)} & =\eta_{\Phi}+\hat{\xi} \in \mathfrak{e}(L) \\
{\left[\eta_{\left(\Phi_{1}, \xi_{1}\right)}, \eta_{\left(\Phi_{2}, \xi_{2}\right)}\right] } & =\eta_{\left(\xi_{1} \Phi_{2}-\xi_{2} \Phi_{1}+\omega\left(\xi_{1}, \xi_{2}\right),\left[\xi_{1}, \xi_{2}\right]\right)}
\end{aligned}
$$

Proof. Let us denote by $\eta_{(\Phi, \xi)}$ the commutator $\left[\eta_{\left(\Phi_{1}, \xi_{1}\right)}, \eta_{\left(\Phi_{2}, \xi_{2}\right)}\right]$. Then

$$
\pi_{*}\left[\eta_{\left(\Phi_{1}, \xi_{1}\right)}, \eta_{\left(\Phi_{2}, \xi_{2}\right)}\right]=\left[\xi_{1}, \xi_{2}\right] \equiv \xi
$$


and

$$
\left\langle\alpha,\left[\eta_{\left(\Phi_{1}, \xi_{1}\right)}, \eta_{\left(\Phi_{2}, \xi_{2}\right)}\right]\right\rangle \equiv-\pi^{*} \Phi
$$

With the identity

$$
\left\langle\alpha,\left[\eta_{1}, \eta_{2}\right]\right\rangle=\mathrm{L}_{\eta_{1}}\left\langle\alpha, \eta_{2}\right\rangle-\mathrm{L}_{\eta_{2}}\left\langle\alpha, \eta_{1}\right\rangle-\mathrm{d} \alpha\left(\eta_{1}, \eta_{2}\right)
$$

(19) becomes

$$
\Phi=\mathrm{L}_{\xi_{1}} \Phi_{2}-\mathrm{L}_{\xi_{2}} \Phi_{1}+\omega\left(\xi_{1}, \xi_{2}\right)
$$

The elements of the algebra $\mathfrak{e}(L)$ act on functions on $L^{*}$, but we can also find a representation of $\mathfrak{e}(L)$ in the space of the sections $\Gamma_{L}$. Thus, we can define an $\mathfrak{e}(L)$-isomorphism $: \Gamma_{L}(M) \mapsto \mathcal{F}\left(L^{*}\right)$ associating to any section $s \in \Gamma_{L}(M)$ a function $\tilde{s} \in \mathcal{F}\left(L^{*}\right)$,

$$
\tilde{s}(x)=\frac{s(\pi x)}{x}, \quad x \in L^{*} .
$$

Proposition 3. If $(\Phi, \xi) \in \mathcal{F}_{c}(M) \times \chi(M)$ then $\eta_{(\Phi, \xi)} \tilde{s}=\tilde{t}$, where

$$
t=\left(\nabla_{\xi}+2 \pi \mathrm{i} \Phi\right) s \equiv \hat{\eta} s .
$$

With respect to a local system $\left\{\left(U_{i}, s_{i}\right), i \in I\right\}$ on $L$, the elements of $\Gamma_{L}$ are represented by functions $\psi_{i}: U_{i} \mapsto \mathbb{C}$, provided by

$$
\left.s\right|_{U_{i}}=\psi_{i} s_{i}, \quad s \in \Gamma_{L} .
$$

For this local trivialization, the operator $\hat{\eta}_{(\Phi, \xi)}=\nabla_{\xi}+2 \pi \mathrm{i} \Phi$ determines an operator $\hat{\eta}_{i(\Phi, \xi)}$ on $\mathcal{F}_{c}\left(U_{i}\right)$

$$
\hat{\eta}_{i(\Phi, \xi)}=\mathrm{L}_{\xi}+2 \pi \mathrm{i}\left(\left\langle\alpha_{i}, \xi\right\rangle+\Phi\right) .
$$

The tangent fields to $L$ which preserve the connection and the Hermitian structure form a subalgebra in $\mathfrak{e}(L)$, denoted $\mathfrak{e}(L, \alpha)$. It can be proved [14] that if $\eta_{(\Phi, \xi)} \in \mathfrak{e}(L)$, then

$$
\mathrm{L}_{\eta_{(\Phi, \xi)}}|*|^{2}=0 \quad \text { iff } \Phi \in \mathcal{F}(M)
$$

and

$$
\mathrm{L}_{\eta_{(\Phi, \xi)}} \alpha=\pi^{*}\left(i_{\xi} \omega-\mathrm{d} \Phi\right)
$$

This shows that

$$
\eta_{(\Phi, \xi)} \in \mathfrak{e}(L, \alpha) \quad \text { iff } \Phi \in \mathcal{F}(M) \text { and } i_{\xi} \omega=\mathrm{d} \Phi .
$$


Therefore, the mapping

$$
\delta: \mathcal{F}(M) \mapsto \mathfrak{e}(L, \alpha) ; \delta(\Phi)=\eta_{\left(\Phi, \xi_{\Phi}\right)}
$$

(or $\mathcal{F}(M) \mapsto \mathfrak{e n d}\left(\Gamma_{L}\right)$ ), called map of prequantiztion, is an isomorphism of Lie algebras.

These results indicate that we can obtain a representation of the Lie algebra of the observables, $\mathcal{F}(M)$, in the prequantum Hilbert space $\mathcal{H}$. In this representation each function $\Phi$ has an associated operator

$$
\hat{\eta}_{\left(\Phi, \xi_{\Phi}\right)}=\nabla_{\xi_{\Phi}}+2 \pi \mathrm{i} \Phi
$$

on $\Gamma_{L}$, or on the space of the local representatives $\psi_{i}$ of the sections,

$$
\hat{\eta}_{i\left(\Phi, \xi_{\Phi}\right)}=\mathrm{L}_{\xi_{\Phi}}+2 \pi \mathrm{i}\left(\left\langle\alpha_{i}, \xi_{\Phi}\right\rangle+\Phi\right) .
$$

Thus, defining the local operator associated to the observable $f$ as

$$
\hat{f}=\frac{1}{2 \pi \mathrm{i}} \hat{\eta}_{\left(f, \xi_{f}\right)} \equiv \frac{1}{2 \pi \mathrm{i}} \mathrm{L}_{\xi_{f}}+\left\langle\alpha_{i}, \xi_{f}\right\rangle+f
$$

one obtains a map which satisfies the conditions 1,2,3 stated in the Section 1 , discussed in detail in [6]. In particular, if $M=\mathbb{R}^{2}, \alpha_{i}=-p \mathrm{~d} q$, then $\xi_{p}=\partial_{q}, \xi_{q}=-\partial_{p}$, and $\hat{p}=-\mathrm{i} \hbar \partial_{q}, \hat{q}=q+\mathrm{i} \hbar \partial_{p}$.

\subsection{The Prequantization of Classical Dynamical Systems}

The classical dynamical systems on the phase-space $(M, \omega)$ are subgroups of $\mathcal{D}(M)$, the group of diffeomorphisms on $M$. The symplectic diffeomorphisms form a subgroup denoted $\mathcal{D}(M, \omega)$, of diffeomorphisms which act by canonical transformations,

$$
\mathcal{D}(M, \omega)=\left\{\rho \in \mathcal{D}(M) ; \rho^{*} \omega=\omega\right\} .
$$

This subgroup contains $\operatorname{Ham}(M, \omega)$, the subgroup of Hamiltonian diffeomorphisms, and if $M$ is simply connected, or if $T M=[T M, T M]$, then $\mathcal{D}(M, \omega)=\operatorname{Ham}(M, \omega)$.

The phase-space $(M, \omega)$ has also an associated set of equivalence classes of Hermitian line bundles with connection, $\mathcal{L}_{c}(M, \omega)$. The group $\mathcal{D}(M, \omega)$ acts on $\mathcal{L}_{c}(M, \omega)$, but prequantum representations in a class $\ell \in \mathcal{L}_{c}(M, \omega)$, can be obtained only for the elements of the stability group $\mathcal{D}_{\ell}(M, \omega)$ of $\ell$ with respect to the action of $\mathcal{D}(M, \omega)$,

$$
\mathcal{D}_{\ell}(M, \omega)=\left\{\rho \in \mathcal{D}(M, \omega) ; \rho_{\mathcal{L}}^{*} \ell=\ell, \ell \in \mathcal{L}_{c}(M, \omega)\right\} .
$$


Thus, if $[L]=\ell$ and $\rho \in \mathcal{D}_{\ell}(M, \omega)$, then $\rho_{\mathcal{L}}^{*} L$ and $L$ are equivalent, and there exists an equivalence of line bundles with connection $\epsilon: \rho_{\mathcal{L}}^{*} L \mapsto L$, uniquely specified up to a phase factor.

In general, if $G$ is a group acting on $(M, \omega)$ by canonical transformations, there are operators $\hat{g}: \mathcal{H} \mapsto \mathcal{H}$ which define a projective representation of $G$ in $\mathcal{H}$, such that for $g_{1}, g_{2} \in G$,

$$
\hat{g}_{1} \hat{g}_{2}=\tau_{12} \hat{g}_{1} \hat{g}_{2}, \quad\left|\tau_{12}\right|=1 .
$$

In the prequantum Hilbert space $\mathcal{H} \subset \Gamma_{L},[L]=\ell$, the operator associated to $\rho \in \mathcal{D}_{\ell}(M, \omega)$ is defined up to a phase factor by the equality

$$
\left(\hat{\rho}^{-1} s\right)_{(p)}=\epsilon\left(\rho^{*} s_{(p)}\right) .
$$

If $\rho_{h}(t) \in \operatorname{Ham}(M, \omega)$ is generated by the Hamiltonian $h$, then $\rho^{*} L=L$ and the operator $\hat{\rho}_{t}: \Gamma_{L} \mapsto \Gamma_{L}$ will be defined by

$$
\tilde{\rho}_{\eta}(t) \circ \hat{\rho}_{t}^{-1} s=s \circ \rho_{h}(t)
$$

where $\tilde{\rho}_{\eta}(t)$ is the group of one-parameter diffeomorphisms on $L^{*}$ determined by $\eta_{\left(h, X_{h}\right)} \in \mathfrak{e}(L, \alpha)$.

Theorem 1. $\eta_{\left(h, X_{h}\right)} \in \mathfrak{e}(L, \alpha)$ is globally integrable on $L^{*}$ iff $X_{h}$ is globally integrable on $M$, and the diagram

$$
\begin{array}{rccc}
\tilde{\rho}_{\eta}(t): & L^{*} & \mapsto & L^{*} \\
& \pi \downarrow & & \pi \downarrow \\
\rho_{t}: & M & \mapsto & M
\end{array}
$$

commutes.

To obtain explicitly the operator $\hat{\rho}_{t}$, we can write $(27)$ in local coordinates. Let $\left\{\left(U_{i}, s_{i}\right), i \in I\right\}$ be a local system, and $\sigma$ the diffeomorphism

$$
\sigma: \mathbb{C} \times U_{i} \mapsto \pi^{-1}\left(U_{i}\right), \quad \sigma(z, p)=z s_{i}(p) .
$$

The functions $\tilde{s}(x) \equiv s(\pi x) / x$ on $L^{*}$ associated to the sections $s \in \Gamma_{L}\left(U_{i}\right)$ are represented locally by functions $\tilde{s}^{b}$ on $\mathbb{C} \times U_{i}$,

$$
\tilde{s}^{b}(z, p)=\tilde{s}\left(z s_{i}(p)\right)=\frac{1}{z} \tilde{s}\left(s_{i}(p)\right)=\frac{1}{z} \psi(p) .
$$

Also, the connection form $\alpha$ and the field $\eta_{(\Phi, \xi)}$ have the local expressions

$$
\alpha^{b}=\alpha_{i}+\frac{1}{2 \pi \mathrm{i}} \frac{\mathrm{d} z}{z}, \quad \alpha_{i}=s_{i}^{*} \alpha
$$




$$
\eta^{b}=\xi^{b}-2 \pi \mathrm{i} \Phi z \partial_{z}
$$

where $\xi^{b}=\xi-2 \pi \mathrm{i}\left(\left\langle\alpha_{i}, \xi\right\rangle z \partial_{z}-\left\langle\bar{\alpha}_{i}, \xi\right\rangle \bar{z} \partial_{\bar{z}}\right) \in \operatorname{ker}\left(\alpha^{b}\right)$. The flow of $\eta^{b}$ determines the time-evolution of the functions $\tilde{s}_{i}^{b}$ by the equation

$$
\frac{\mathrm{d} \tilde{s}_{i}^{b}}{\mathrm{~d} t}=\eta^{\mathrm{b}} \tilde{s}_{i}^{b}
$$

This provides the dependence on time of the coordinate $z$ and of the point $p \in U_{i}$ in terms of the local expression, denoted $\tilde{\rho}_{\eta^{b}}^{b}(t)$,

$$
\tilde{\rho}_{\eta^{b}}^{b}(t)\left(z_{0}, p_{0}\right)=\left(z_{0} \mathrm{e}^{-2 \pi \mathrm{i} \int_{0}^{t} d t^{\prime}\left(\left\langle\alpha_{i}, \xi\right\rangle+\Phi\right)}, \rho_{t}\left(p_{0}\right)\right)
$$

of the flow $\tilde{\rho}_{\eta}(t)$.

The operator $\hat{\rho}(t)$ defines an operator $\hat{U}_{t}$ acting on the complex functions $\psi(p)=s(p) / s_{i}(p)$, representing the sections $s \in \Gamma_{L}\left(U_{i}\right)$, by

$$
\hat{U}_{t} \psi=\frac{\hat{\rho}_{t} s}{s_{i}}
$$

Explicitly, this is obtained from (27) in local form,

$$
\tilde{\rho}_{\eta}^{b}(t)\left(\hat{U}_{t}^{-1} \psi, p\right)=\left(\psi\left(\rho_{t} p\right), \rho_{t}(p)\right)
$$

where the action of $\tilde{\rho}_{\eta}^{b}$ is given by $(31)$,

$$
\tilde{\rho}_{\eta}^{b}(t)\left(\hat{U}_{t}^{-1} \psi, p\right)=\left(\mathrm{e}^{-2 \pi \mathrm{i} \int_{0}^{t} \mathrm{~d} t^{\prime}\left(\left\langle\alpha_{i}, \xi\right\rangle+\Phi\right)}\left(\hat{U}_{t}^{-1} \psi\right)_{p}, \rho_{t}(p)\right) .
$$

The result

$$
\left(\hat{U}_{t}^{-1} \psi\right)_{p}=\mathrm{e}^{2 \pi \mathrm{i} \int_{0}^{t} \mathrm{~d} t^{\prime}\left(\left\langle\alpha_{i}, \xi\right\rangle+\Phi\right)} \psi\left(\rho_{t}(p)\right)
$$

agrees with the expression derived in the previous subsection for the local operator

$$
\hat{\Phi}=\frac{1}{2 \pi \mathrm{i}} \mathrm{L}_{\xi_{\Phi}}+\left\langle\alpha_{i}, \xi_{\Phi}\right\rangle+\Phi
$$

because

$$
\mathrm{i} \hbar \frac{\mathrm{d}}{\mathrm{d} t}\left(\hat{U}_{t} \psi\right)=\hat{\Phi} \hat{U}_{t} \psi
$$




\subsection{Applications to Elementary Systems}

Let $G$ be a simply connected Lie group with the Lie algebra $\mathfrak{g}$, and $\mathfrak{g}^{*}$ the dual of $\mathfrak{g}$. For $f \in \mathfrak{g}^{*}$ one can define on $G$ a right $\left(R_{g}\right)$ - invariant 1 -form $\theta_{f}$, and a closed 2-form $\omega_{f}=\mathrm{d} \theta_{f}$,

$$
\left.\omega_{f}(x, y)\right|_{e}=\langle f,[x, y]\rangle, \quad x, y \in \mathfrak{g} .
$$

The distribution $K_{f}$ determined by the kernel of $\omega_{f}$ on $G$ has as tangent space at the identity $e$

$$
T_{e} K_{f}=\{x \in \mathfrak{g} ;\langle f,[x, y]\rangle=0 \text { for any } y \in \mathfrak{g}\}
$$

namely the algebra $\mathfrak{g}_{f}$ of the stability group $G_{f}$ of $f$ with respect to the coadjoint action of $G$. Thus, the leaf of $K_{f}$ through $e$ is the connected component $\left(G_{f}\right)_{0}$ of $G_{f}$, and if closed, $M^{\prime}=G / K_{f} \simeq G /\left(G_{f}\right)_{0}$ is covering space for the orbit $M_{f}=G / G_{f}$ of $f$ in $\mathfrak{g}^{*}$.

Theorem 2. Let $\left(M^{\prime}, \omega^{\prime}\right)$ be the reduced phase-space associated to the reducible presymplectic manifold $\left(G, \omega_{f}\right)$. Then $\left(M^{\prime}, \omega^{\prime}\right)$ is quantizable iff $f$ can be integrated to a character for $\left(G_{f}\right)_{0}$.

Proof. Let us assume first that

$$
\oint_{\gamma} \theta_{f} \in \mathbb{Z}
$$

(the BWS condition) with $\gamma \subset\left(G_{f}\right)_{0}$. Thus, one can define

$$
\chi_{f}(h)=\mathrm{e}^{2 \pi \mathrm{i} \int_{e}^{h} \theta_{f}}
$$

where the integral can be taken along any curve in $\left(G_{f}\right)_{0}$, which joins $e$ to $h$. Because

$$
\chi_{f}\left(h_{1} h_{2}\right)=\mathrm{e}^{2 \pi \mathrm{i} \int_{e}^{h_{1} h_{2}} \theta_{f}}=\mathrm{e}^{2 \pi \mathrm{i} \int_{e}^{h_{2}} \theta_{f}+2 \pi \mathrm{i} \int_{h_{2}}^{h_{1} h_{2}} \theta_{f}}
$$

independently of the integration path, from the BWS condition, while

$$
\int_{h_{2}}^{h_{1} h_{2}} \theta_{f}=\int_{e}^{h_{1}} \theta_{f}
$$

from the $R_{g}$-invariance of $\theta_{f}$, one obtains

$$
\chi_{f}\left(h_{1} h_{2}\right)=\chi_{f}\left(h_{1}\right) \chi_{f}\left(h_{2}\right)
$$


such that $\chi_{f}$ is a character for $\left(G_{f}\right)_{0}$. If $h=\mathrm{e}^{t x}$, with $x \in \mathfrak{g}_{f}$, then

$$
\chi_{f}\left(\mathrm{e}^{t x}\right)=\mathrm{e}^{2 \pi \mathrm{i}\langle f, x\rangle t}
$$

from the $R_{g}$-invariance, such that $f$ appears as an infinitesimal character in the sense that

$$
\begin{aligned}
\left.\frac{\mathrm{d}}{\mathrm{d} t} \chi_{f}\left(\mathrm{e}^{t x}\right)\right|_{t=0} & =2 \pi \mathrm{i}\langle f, x\rangle, \quad x \in \mathfrak{g}_{f} \\
\langle f,[x, y]\rangle & =0, \quad x, y \in \mathfrak{g}_{f} .
\end{aligned}
$$

Conversly, the condition to integrate the infinitesimal character $f$ to a character of $\left(G_{f}\right)_{0}$ independently of the path, applied to (37) derived from (40), leads to the BWS condition $\square$.

Let us consider

$$
G=\mathrm{SU}(2)=\left\{\left[\begin{array}{cc}
z_{0} & z_{1} \\
-\bar{z}_{1} & \bar{z}_{0}
\end{array}\right] ;\left|z_{0}\right|^{2}+\left|z_{1}\right|^{2}=1\right\} \simeq \mathbb{S}^{3} \subset \mathbb{C}^{2} .
$$

The algebra $\mathfrak{g} \equiv T_{e} G$ of $G$ consists of matrices

$$
x_{a}=-\frac{\mathrm{i}}{2}\left[\begin{array}{cc}
a_{1} & a_{2}-\mathrm{i} a_{3} \\
a_{2}+\mathrm{i} a_{3} & -a_{1}
\end{array}\right]=\sum_{i=1}^{3} a_{i} E_{i}
$$

with $\left(a_{1}, a_{2}, a_{3}\right) \equiv \mathbf{a} \in \mathbb{R}^{3}$ and $\left[E_{i}, E_{j}\right]=\epsilon_{i j k} E_{k}$.

The right (left) - invariant vector fields $Y_{a}\left(Z_{a}\right)$ extending $x_{a} \in \mathfrak{g}$ are $^{4}$

$$
\begin{aligned}
Y_{a} & =-\frac{\mathrm{i}}{2}\left[\left(a_{1} z_{0}-\left(a_{2}-\mathrm{i} a_{3}\right) \bar{z}_{1}\right) \partial_{z_{0}}+\left(a_{1} z_{1}+\left(a_{2}-\mathrm{i} a_{3}\right) \bar{z}_{0}\right) \partial_{z_{1}}\right]+c . c . \\
Z_{a} & =-\frac{\mathrm{i}}{2}\left[\left(a_{1} z_{0}+\left(a_{2}+\mathrm{i} a_{3}\right) z_{1}\right) \partial_{z_{0}}+\left(-a_{1} z_{1}+\left(a_{2}-\mathrm{i} a_{3}\right) z_{0}\right) \partial_{z_{1}}\right]+c . c .
\end{aligned}
$$

and the right-invariant 1 -form $\theta_{f}$ associated to $f \in \mathfrak{g}^{*} \simeq \mathfrak{g}$ is

$$
\theta_{f}=\mathrm{i}\left[\left(f_{1} \bar{z}_{0}-\left(f_{2}+\mathrm{i} f_{3}\right) z_{1}\right) \mathrm{d} z_{0}+\left(f_{1} \bar{z}_{1}+\left(f_{2}+\mathrm{i} f_{3}\right) z_{0}\right) \mathrm{d} z_{1}\right]+\text { c.c. }
$$

where c.c. is the complex conjugate of the previous term. In particular, for $\mathbf{f} \equiv\left(f_{1}, f_{2}, f_{3}\right)=(-l, 0,0)$ we get

$$
\left(G_{f}\right)_{0}=G_{f}=\left\{\left[\begin{array}{cc}
\mathrm{e}^{\mathrm{i} t} & 0 \\
0 & \mathrm{e}^{-\mathrm{i} t}
\end{array}\right] ; t \in \mathbb{R}\right\} \subset G
$$

\footnotetext{
${ }^{4}$ If $x_{a}, x_{b} \in \mathfrak{g}$ and $\left[x_{a}, x_{b}\right]=x_{c}$, then $\left[Z_{a}, Z_{b}\right]=Z_{c},\left[Y_{a}, Y_{b}\right]=-Y_{c}$.
} 
and

$$
\theta_{f}=\mathrm{i} l \sum_{k=0}^{1}\left(z_{k} \mathrm{~d} \bar{z}_{k}-\bar{z}_{k} \mathrm{~d} z_{k}\right), \quad \omega_{f}=2 \mathrm{i} l \sum_{k=0}^{1} \mathrm{~d} z_{k} \wedge \mathrm{d} \bar{z}_{k} .
$$

Each $h_{t} \in G_{f}$ is generated by $x_{a} \in \mathfrak{g}$ with $\mathbf{a}=(-2,0,0)$,

$$
x_{a}=\left[\begin{array}{cc}
\mathrm{i} & 0 \\
0 & -\mathrm{i}
\end{array}\right], \quad h_{t}=\mathrm{e}^{t x_{a}}=\left[\begin{array}{cc}
\mathrm{e}^{\mathrm{i} t} & 0 \\
0 & \mathrm{e}^{-\mathrm{i} t}
\end{array}\right]
$$

and as

$$
\left\langle\theta_{f}, Y_{a}\right\rangle=\left\langle f, x_{a}\right\rangle=\mathbf{f} \cdot \mathbf{a}=-a_{1} l=2 l
$$

it determines a character

$$
\chi_{f}\left(h_{t}\right)=\mathrm{e}^{2 \pi \mathrm{i}\left\langle\theta_{f}, Y_{a}\right\rangle t}=\mathrm{e}^{4 \pi \mathrm{i} l t} .
$$

This character allows one to define a line bundle $L^{\prime}$ on $M^{\prime}=G / G_{f}$ by factorizing the trivial bundle $G \times \mathbb{C}$ with respect to the equivalence relation " ",

$$
(g, z) \sim\left(h g, \chi_{f}(h) z\right)
$$

where $g \in G, h \in G_{f}$ and $z \in \mathbb{C}$. The sections in $\Gamma_{L^{\prime}}$ are represented by functions $\psi: G \mapsto \mathbb{C}$ (sections in $G \times \mathbb{C}$ ) which satisfy the global relation

$$
\psi(h g)=\chi_{f}(h) \psi(g)
$$

or locally

$$
Y_{a} \psi(g)=2 \pi \mathbf{i} \mathbf{a} \cdot \mathbf{f} \psi(g) .
$$

Thus, the sections of $\Gamma_{L^{\prime}}$ are represented in the coordinates $\left(z_{i}, \bar{z}_{i}\right)$ by functions $\psi: \mathbb{S}^{3} \mapsto \mathbb{C}$ which satisfy

$$
\sum_{k=0}^{1}\left(z_{k} \partial_{z_{k}}-\bar{z}_{k} \partial_{\bar{z}_{k}}\right) \psi(z, \bar{z})=4 \pi l \psi(z, \bar{z})
$$

The equivalence relation " $\sim$ " is well defined, and $M^{\prime}$ is quantizable if

$$
\chi_{f}\left(h_{2 \pi}\right)=\mathrm{e}^{8 \pi^{2} i l}=1, \quad 4 \pi l \in \mathbb{Z}
$$

namely $l=n \hbar / 2$ (here $\hbar=1 / 2 \pi$ ), with $n \in \mathbb{Z}$. A physical application to the intrinsic angular momentum (spin) is presented in [24].

The points of the phase-space $M^{\prime}$ correspond to equivalence classes in $G$ defined by

$$
[g]=\left\{h g ; h \in G_{\mathbf{f}}, g \in G\right\} .
$$


Let $\operatorname{pr}: G \mapsto M^{\prime}$ be the projection $\operatorname{pr}(g)=[g], g \in G$. A canonical action

$$
g_{1}[g]=\left[g g_{1}^{-1}\right]
$$

of $G$ on $M^{\prime}$ can be defined by the projection on $M^{\prime}$ of the action to the right of $G$ on $G$ (because the equivalence necessary for projection is obtained by the action to the left), and $M^{\prime}$ becomes a homogeneous phase-space for $G$. Locally, the action of $G$ on $M^{\prime}$ arises by the projection of the leftinvariant fields, $-Z_{a}$, on $T M^{\prime}, \operatorname{pr}_{*}\left(-Z_{a}\right)=X_{a}$, and because the algebra $\mathfrak{g}$ is semisimple, there exists a lift $\lambda$ of this action such that the diagram

$$
\begin{aligned}
0 \mapsto \mathcal{F}\left(M^{\prime}\right) & \mapsto \mathfrak{h a m}\left(M^{\prime}\right) \quad \mapsto 0 \\
\lambda \nwarrow \uparrow & \mapsto{ }^{\prime}
\end{aligned}
$$

$\mathfrak{g}$

commutes. Explicitly, for any $x_{a} \in \mathfrak{g}$ one can find $h_{a}: M^{\prime} \mapsto \mathbb{C}, \mathrm{pr}^{*} h_{a}=$ $\left\langle\theta_{\mathbf{f}}, Z_{a}\right\rangle$, representing the Hamiltonian of the field $X_{a}$,

$$
i_{X_{a}} \omega^{\prime}=\mathrm{d} h_{a} .
$$

To get the time-evolution of the sections from the line bundle $L^{\prime}$, associated with the dynamical system generated on $M^{\prime}$ by the Hamiltonian $h_{a}$, we project on $L^{\prime}$ the trajectory in $G \times \mathbb{C}$ of the dynamical system generated by the Hamiltonian $\left\langle\theta_{f}, Z_{a}\right\rangle$. This trajectory is given by (35) in which

$$
\begin{gathered}
\alpha_{i}=\theta_{f}, \quad \xi=-Z_{a}, \quad \Phi=\left\langle\theta_{f}, Z_{a}\right\rangle \\
\left(\hat{U}_{g_{t}} \psi\right)(g)=\psi\left(g g_{t}\right), \quad g \in G
\end{gathered}
$$

while the projection on $\Gamma_{L^{\prime}}$ requires $\psi$ constrained by (47),

$$
\psi(h g)=\chi_{f}(h) \psi(g), \quad g \in G, \quad h \in G_{f} .
$$

The result indicates that the prequantization of the phase-space $\left(M^{\prime}, \omega^{\prime}\right)$ is equivalent to the derivation of the representations of the group $G$ induced by the character $\chi_{f}$ of the subgroup $G_{f}$ [15]. In general these representations are not irreducible (do not provide a quantization for $\left(M^{\prime}, \omega^{\prime}\right)$ ), but imposing the condition as $\psi$ to be holomorphic, we obtain irreducible representations. Thus, the holomorphy condition, by introducing a complex polarization, represents a way of restricting the prequantum Hilbert space.

The technique of the induced representations was successfully applied to quantize the relativistic free particle or the liquid drop. In both cases 
the classical configuration space is the orbit of a group $H$ in a linear space $V$, and the quantization consists of finding induced representations for the semidirect product $G=H \times V$. In the first case $H=\mathrm{O}(3,1)$ is the Lorentz group, $V=\mathbb{R}^{3,1}$ is the Minkowski space, and $G$ is the Poincaré group, while in the second case $H=\mathrm{SL}(3, \mathbb{R}), V=\mathbb{R}^{4}$, and $G=\mathrm{CM}(3)[23,22,20]$. The case of a massive free particle in the anti-de Sitter spacetime is considered in $[2]$.

\section{Elements of Quantization}

\subsection{Complex Polarizations}

A complex polarization of the $2 n$-dimensional manifold $(M, \omega)$ is a complex distribution $P$ having the following properties:

i) for any $m \in M, P_{m} \subset T_{m}^{c} M$ is a complex Lagrangian subspace.

ii) $D_{m}=P_{m} \cap \bar{P}_{m} \cap T_{m} M$ has a constant dimension.

iii) $P$ is integrable, in the sense that for any $m \in M$ there exists a collection of functions $\left\{z_{k} \in \mathcal{F}_{c}(M), k=1, n\right\}$, such that $\left\{\bar{X}_{z_{k}}, k=1, n\right\}$ generate $P_{m}$.

Let us introduce the notation

$$
\begin{gathered}
\chi_{c}(U, P)=\left\{X \in \chi_{c}(U) ; X_{m} \in P_{m}, \quad m \in U \subset M\right\} \\
\mathcal{F}_{c}(U, P)=\left\{f \in \mathcal{F}_{c}(U) ; \bar{X} f=0, \quad X \in \chi_{c}(U, P)\right\} \\
=\left\{f \in \mathcal{F}_{c}(U) ; \bar{X}_{f} \in \chi_{c}(U, P)\right\} \\
\mathcal{F}_{c}(U, P, 1)=\left\{f \in \mathcal{F}_{c}(U) ;\{f, g\} \in \mathcal{F}_{c}(U \cap V, P), V \subset M, g \in \mathcal{F}_{c}(V, P)\right\} .
\end{gathered}
$$

The set $\mathcal{F}_{c}(U, P, 1)$ consists of functions having the property that generate flows which preserve the polarization,

$$
\mathrm{L}_{X_{f}} \bar{P} \subset \bar{P} \Leftrightarrow f \in \mathcal{F}_{c}(U, P, 1) .
$$

When $f \in \mathcal{F}_{c}(U, P, 1)$ is real, the flow of $X_{f}$ preserves both $\bar{P}$ and $\omega$. The polarization $P$ is called admisible if on a neighborhood of any $m \in M$, there exists a symplectic potential $\beta$, which is adapted to $P$ in the sense that

$$
i_{\bar{X}} \beta=0, \quad X \in \chi_{c}(M, P) .
$$

The polarization $P$ is of Kähler type if $P_{m} \cap \bar{P}_{m}=\{0\}$ and $T_{m} M=P_{m}+\bar{P}_{m}$. In the Kähler case any $X \in T_{m} M$ can be written as $X=Z+\bar{Z}$, with $Z \in P_{m}$, and $T_{m} M$ carries a complex structure,

$$
J_{m}: T_{m} M \mapsto T_{m} M, \quad J_{m} X=\mathrm{i} Z-\mathrm{i} \bar{Z}
$$


compatible with the symplectic form $\omega$ in the sense that $\omega(J X, J Y)=$ $\omega(X, Y)$.

Let $(M, \omega, J)$ be a Kähler manifold (Appendix [10]), and $\left\{z_{k}, k=1, n\right\}$ local complex coordinates such that

$$
J \partial_{z_{k}}=\mathrm{i} \partial_{z_{k}}, \quad J \partial_{\bar{z}_{k}}=-\mathrm{i} \partial_{\bar{z}_{k}} .
$$

On $M$ can be introduced two polarizations: the holomorphic polarization $P$ generated at any point by the vectors $\left\{\partial_{z_{k}}, k=1, n\right\}$, and the antiholomorphic polarization $\bar{P}$ generated by $\left\{\partial_{\bar{z}_{k}}, k=1, n\right\}$. Thus, for any $U \subset M$,

$$
\mathcal{F}_{c}(U, P)=\{f: U \mapsto \mathbb{C} ; f=\text { holomorphic }\} .
$$

\subsection{Phase-Space Quantization for Kähler Polarizations}

Let $(M, \omega)$ be a symplectic manifold, $\left\{q_{k}, p_{k}, k=1, n\right\}$ the local canonical coordinates for $\omega\left(\omega=\sum_{k=1}^{n} \mathrm{~d} q_{k} \wedge \mathrm{d} p_{k}\right)$, and $P$ a Kähler polarization on $M$ locally generated by the vectors

$$
\left\{\partial_{z_{k}} ; z_{k}=\left(q_{k}-\mathrm{i} p_{k}\right) / \sqrt{2}, k=1, n\right\} .
$$

The complex potential adapted to $P$ is

$$
\beta=\mathrm{i} \sum_{k=1}^{n} \bar{z}_{k} \mathrm{~d} z_{k}
$$

and $\omega=\mathrm{d} \beta$. If $(M, \omega)$ is quantizable, then there exists a Hermitian line bundle with connection $(L, \alpha)$ on $M$, with the sections space $\Gamma_{L}(M)$ and associated prequantum Hilbert space $\mathcal{H}$. We can further define the space of polarized sections

$$
\Gamma_{L}(M, P)=\left\{s \in \Gamma_{L}(M) ; \nabla_{\bar{X}} s=0, \quad X \in \chi_{c}(M, P)\right\}
$$

and the quantum Hilbert space $\mathcal{H}_{P}=\mathcal{H} \cap \Gamma_{L}(M, P)$. The space $\Gamma_{L}(M, P)$ is well defined because the local integrability condition for the sections $s \in$ $\Gamma_{L}(M, P)$ is satisfied. Thus, if

$$
\nabla_{\bar{X}} s=\nabla_{\bar{Y}} s=0, \quad X, Y \in \chi_{c}(M, P)
$$

then

$$
\nabla_{[\bar{X}, \bar{Y}]} s=\left[\nabla_{\bar{X}}, \nabla_{\bar{Y}}\right] s-2 \pi \mathrm{i} \omega(\bar{X}, \bar{Y}) s=0
$$


because $\omega(\bar{X}, \bar{Y})=0$.

The Hilbert space $\mathcal{H}_{P}$ is not invariant to the action of any operator associated with a classical observable, and one should specify which classical observables provide operators on $\mathcal{H}_{P}$. If $f \in \mathcal{F}(M)$, then

$$
\hat{f}=\frac{1}{2 \pi \mathrm{i}} \nabla_{X_{f}}+f
$$

and the condition $\hat{f} \mathcal{H}_{P} \subset \mathcal{H}_{P}$ yields

$$
\nabla_{\bar{X}} \hat{f} s=0, \quad X \in \chi_{c}(M, P), \quad s \in \mathcal{H}_{P} .
$$

However, because

$$
\begin{gathered}
2 \pi \mathrm{i} \nabla_{\bar{X}} \hat{f} s=\nabla_{\bar{X}}\left(\nabla_{X_{f}}+2 \pi \mathrm{i} f\right) s=\left(\left[\nabla_{\bar{X}}, \nabla_{X_{f}}\right]+2 \pi \mathrm{i} L_{\bar{X}} f\right) s \\
=\left(\nabla_{\left[\bar{X}, X_{f}\right]}+2 \pi \mathrm{i} \omega\left(\bar{X}, X_{f}\right)+2 \pi \mathrm{i}_{\bar{X}} f\right) s=\nabla_{\left[\bar{X}, X_{f}\right]} s
\end{gathered}
$$

the condition (57) is equivalent to $\mathrm{L}_{X_{f}} \bar{P} \subset \bar{P}$. Thus, an observable $f$ determines an operator on $\mathcal{H}_{P}$ only if $f \in \mathcal{F}_{c}(M, P, 1)$.

Let $s$ be a section of $\Gamma_{L}(M, P)$ for which $s^{*} \alpha=\beta$, and $r$ the unit section in $\Gamma_{L}(U)$ such that $r^{*} \alpha=\sum_{k=1}^{n} q_{k} \mathrm{~d} p_{k}$. Then, $s=\mathrm{e}^{\varphi} r$, with $\varphi$ specified (up to an additive constant $\varphi_{0}$ ) by

$$
\nabla_{X} s=2 \pi \mathrm{i}\langle\beta, X\rangle s=\nabla_{X}\left(\mathrm{e}^{\varphi} r\right)=\left(\mathrm{L}_{X} \varphi\right) s+2 \pi \mathrm{i}\left\langle r^{*} \alpha, X\right\rangle s
$$

with

$$
\langle\beta, X\rangle=\left\langle r^{*} \alpha, X\right\rangle+\frac{1}{2 \pi \mathrm{i}}\langle\mathrm{d} \varphi, X\rangle, \quad X \in \chi(M)
$$

such that

$$
\begin{aligned}
\mathrm{d} \varphi & =2 \pi \mathrm{i}\left(\beta-r^{*} \alpha\right)=2 \pi \mathrm{i} \sum_{k=1}^{n}\left[\frac{\mathrm{i}}{2}\left(q_{k}+\mathrm{i} p_{k}\right) \mathrm{d}\left(q_{k}-\mathrm{i} p_{k}\right)-q_{k} \mathrm{~d} p_{k}\right] \\
& =-\frac{\pi}{2} \sum_{k=1}^{n} \mathrm{~d}\left(q_{k}^{2}+p_{k}^{2}+2 \mathrm{i} q_{k} p_{k}\right)=-\pi \sum_{k=1}^{n} \mathrm{~d}\left(\left|z_{k}\right|^{2}+\mathrm{i} q_{k} p_{k}\right) .
\end{aligned}
$$

Considering $\varphi_{0}=0$, we get $\varphi=-\pi \sum_{k=1}^{n}\left(\left|z_{k}\right|^{2}+\mathrm{i} p_{k} q_{k}\right)$ and

$$
(s, s)=\mathrm{e}^{-2 \pi \sum_{k=1}^{n}\left|z_{k}\right|^{2}} .
$$

Thus, with respect to the local system specified by $s$, the elements of the space $\mathcal{H}_{P}$ are sections of the form $\left\{\psi_{p} s_{p}, p \in U \subset M\right\}$, where $\psi_{p}$ are 
holomorphic functions of $\left\{z_{k}, k=1, n\right\}$, and the inner product (13) is given by

$$
<\psi_{1}, \psi_{2}>\sim \int \epsilon_{\omega} \bar{\psi}_{1}(z) \psi_{2}(z) \mathrm{e}^{-2 \pi \sum_{k=1}^{n}\left|z_{k}\right|^{2}} .
$$

This Hilbert space coincides with the representation introduced in 1928 by V. Fock, to study the states of the harmonic oscillator. Though, its domain of applicability remains limited because the only observables quantizable in $\mathcal{H}_{P}$ are polynomials in coordinates and momenta of degree at most 2.

For the harmonic oscillator the classical Hamiltonian is $h=2 \pi \nu \bar{z} z$, and

$$
X_{h}=2 \pi \mathrm{i} \nu\left(z \partial_{z}-\bar{z} \partial_{\bar{z}}\right) .
$$

The operator $\hat{h}$ in $\mathcal{H}$ associated with $h$,

$$
\hat{h}=\frac{1}{2 \pi \mathrm{i}} \mathrm{L}_{X_{h}}+\left\langle\beta, X_{h}\right\rangle+h=\nu\left(z \partial_{z}-\bar{z} \partial_{\bar{z}}\right)
$$

becomes $\hat{h}_{P}=\nu z \partial_{z}$ when restricted to $\mathcal{H}_{P}$. Its eigenvalues are $n \nu, n \in \mathbb{Z}$, showing that this approach yields the same result, physically incomplete, as the old quantum mechanics. To obtain the missing term $\nu / 2$ the bundle of polarized sections should be extended by a line bundle of half-forms providing the measure for the inner product in $\mathcal{H}_{P}$, as indicated on examples in [19].

\subsection{Real Polarizations and Asymptotic Solutions}

A real polarization of the symplectic manifold $(M, \omega)$ is a foliation of $M$ by Lagrangian (maximal isotropic) submanifolds. If $M=T^{*} Q$, and $\omega=$ $\sum_{k=1}^{n} \mathrm{~d} q_{k} \wedge \mathrm{d} p_{k}$ is the canonical 2-form, then the vertical foliation $P$ is a real polarization, and the leaves of $P$ are the surfaces $q_{k}=$ constant, $k=1, n$.

Let $P$ be a real polarization of the symplectic manifold $(M, \omega)$. Then, on a neighborhood of any point $m$ of $M$ one can find canonical coordinates $(x, y) \equiv\left(x_{k}, y_{k}\right)_{k=1, n}$ such that the leaves of $P$ coincide locally with the surfaces $x=$ constant or $y=$ constant. The canonical coordinates having this property are called "adapted to $P$ ".

Let $\Lambda \subset M$ be a Lagrangian submanifold and $U \subset M$ containing $\Lambda$ such that $\left.\omega\right|_{U}=\mathrm{d} \theta$. Because $\left.\omega\right|_{\Lambda}=0$ then also $\left.\mathrm{d} \theta\right|_{\Lambda}=0$, and locally there exists a function $\wp$ on $\Lambda$, called "local phase function", $\wp: \Lambda \mapsto \mathbb{R}$, such that $\left.\theta\right|_{\Lambda}=-\mathrm{d} \wp$.

If $M=T^{*} Q$, then $\Lambda$ is transversal to the vertical polarization $P$ if the restriction to $\Lambda$ of the projection 


\section{$\Lambda \subset M$ \\ $\pi \downarrow$ \\ $Q$}

is a diffeomorphism. In this case $S \in \mathcal{F}(W), \pi(\Lambda)=W \subset Q, \pi^{*} S=\wp$, is called "generating function of the first kind" of $\Lambda$. Moreover, $\Lambda \cap T^{*} Q$ determines a 1-form on $W$ with the local coordinates

$$
(p, q) \equiv\left(\frac{\partial S}{\partial q}, q\right)
$$

Thus, a foliation of the phase-space $M=T^{*} Q$ by Lagrangian submanifolds corresponds to a family of generating functions $S(q, y), y \equiv\left\{y_{k}, k=1, n\right\}$, parameterized by the variables $y$. This type of foliation appears naturally in classical mechanics by the Hamilton-Jacobi equation,

$$
h\left(\partial_{q} S, q\right)=\text { constant }
$$

which represents the condition $\left.h\right|_{\Lambda_{S}}=$ constant for the Lagrangian submanifold $\Lambda_{S}$ of $T^{*} Q$ generated by $S$.

Proposition 4. Let $\Lambda \subset M$ be a connected Lagrangian submanifold of the phase-space $(M, \omega)$ and $h \in \mathcal{F}(M)$. Then $h$ is a constant on $\Lambda$ iff $X_{h} \in \chi(M)$ is tangent to $\Lambda$.

If we denote $x \equiv\left\{x_{k}=\partial S / \partial y_{k}, k=1, n\right\}$, then $(x, y)$ is a local coordinate system on $T^{*} Q$ adapted to the polarization $\Lambda_{S}$ determined by $S(q, y)$. In this system $h$ is a function only of $y$, and the equations of motion are

$$
\dot{y}=0, \quad \dot{x}=\text { constant. }
$$

In particular, when $Q \simeq \mathbb{R}^{n}$ and $\Lambda_{S} \subset \mathbb{T}^{n} \equiv \mathbb{R}^{n} / \mathbb{Z}^{n}$ is part of an invariant torus, then $y-x$ are the "action-angle" coordinates used to express the BWS conditions.

To quantize a classical system described by the Hamiltonian $h$ it is convenient to find a Hilbert space $\mathcal{H}_{\Lambda_{S}}$ associated to the polarization determined by the solution $S$ of the Hamilton-Jacobi equation. Because in the case $\Lambda_{S} \subset \mathbb{T}^{n}$ the BWS conditions provide constraints on the phase-space reduction (to a point) and the stationary states, it is natural to select the sections from $\Gamma_{L}\left(M, \Lambda_{S}\right)$ by $\nabla_{X} r=0$, where $X$ is tangent to $\Lambda_{S}$ and $r \in \Gamma_{L}(M)$. Let $s$ be a section in $\Gamma_{L}(M)$ such that

$$
\left.s^{*} \alpha\right|_{U_{i}}=-\mathrm{d} S
$$


and $r=\psi s$ an arbitrary element in $\Gamma_{L}\left(M, \Lambda_{S}\right)$. The equation

$$
\nabla_{X} r=\left(\mathrm{L}_{X} \psi\right) s-2 \pi \mathrm{i}\langle\mathrm{d} S, X\rangle \psi s=\left(\mathrm{L}_{X} \psi\right) s-2 \pi \mathrm{i}\left(\mathrm{L}_{X} S\right) \psi s=0
$$

has the solution $\ln \psi-2 \pi \mathrm{i} S=f(y)$, where $f$ is an arbitrary function of $y$, or

$$
\psi(q, y)=a(y) \mathrm{e}^{2 \pi \mathrm{i} S(q, y)} .
$$

The sections from $\Gamma_{L}\left(M, \Lambda_{S}\right)$ can be transferred to the space $\Gamma_{L}(M, P)$, where $P$ is the vertical polarization associated to the Schrödinger representation. The function obtained [30]

$$
\Psi(q)=A(q) \mathrm{e}^{2 \pi \mathrm{i} S(q)}
$$

can be interpreted as asymptotic solution of the Schrödinger equation in the WKB [27] approximation.

As $h$ may contain any potential, in general $S$ is multiple-valued, and $\Psi$ should be defined by a sum $\sum_{\mu} A_{\mu} \exp \left(2 \pi \mathrm{i} S_{\mu}\right)$ over different branches. Presuming that $A_{\mu}$ can have the branches $\pm\left|A_{\mu}\right|$, but $\Psi$ remains singlevalued, one obtains corrected BWS quantum conditions [13], which yield for the harmonic oscillator the exact energy levels $\nu(n+1 / 2), n=0,1,2, \ldots$.

\section{Quantization and Discretization}

The geometric elements presented in the previous sections also appear in the formalism of statistical mechanics. Let $f \geq 0$ be the distribution function [26] of a classical system composed of $\mathrm{N}$ identical, non-interacting particles, defined on the one-particle phase-space $(M, \omega)$, normalized by

$$
\int_{M} \epsilon_{\omega} \mathrm{f}(\mathbf{q}, \mathbf{p}, t)=\mathrm{N}, \quad \mathrm{N} \geq 1 .
$$

For a one-particle Hamilton function $h: M \mapsto \mathbb{R}$, at zero temperature and without friction, $f(\mathbf{q}, \mathbf{p}, t)$ evolves according to the transport equation

$$
\partial_{t} f+L_{X_{h}} f=0 \text {. }
$$

Let us consider $M=T^{*} \mathbb{R}^{3}, \omega=\sum_{i=1}^{3} \mathrm{~d} q_{i} \wedge \mathrm{d} p_{i}, h(\mathbf{q}, \mathbf{p})=\mathbf{p}^{2} / 2 m+V(\mathbf{q})$, and

$$
\mathbf{f}(\mathbf{q}, \mathbf{p}, t)=\frac{1}{(2 \pi)^{3}} \int \mathrm{d}^{3} k \mathrm{e}^{-\mathrm{ik} \cdot \mathbf{p}} \tilde{\mathbf{f}}(\mathbf{q}, \mathbf{k}, t)
$$


where $\tilde{\mathbf{f}}(\mathbf{q}, \mathbf{k}, t)$ is the Fourier transform of $\mathbf{f}(\mathbf{q}, \mathbf{p}, t)$. In this case, a particular class of exact solutions for $(62)$ are the "action distributions" $\mathbf{f}_{0}(\mathbf{q}, \mathbf{p}, t)$, provided by

$$
\tilde{\mathrm{f}}_{0}(\mathbf{q}, \mathbf{k}, t)=\mathrm{n}(\mathbf{q}, t) \mathrm{e}^{\mathrm{i} \mathbf{k} \cdot \partial_{\mathbf{q}} S(\mathbf{q}, t)}
$$

where $\mathbf{n}(\mathbf{q}, t) \geq 0$ (the particle density) and $S(\mathbf{q}, t)$ satisfy the continuity, respectively the Hamilton-Jacobi equations [8].

The partial derivative $\mathbf{k} \cdot \partial_{\mathbf{q}} S(\mathbf{q}, t)$ in (64) is the limit of

$$
\frac{k}{\ell}\left[S\left(\mathbf{q}+\frac{\ell}{2 k} \mathbf{k}, t\right)-S\left(\mathbf{q}-\frac{\ell}{2 k} \mathbf{k}, t\right)\right]
$$

$k=|\mathbf{k}|$, when $\ell \rightarrow 0$. If a new parameter $\sigma=\ell / k$ is introduced, then

$$
\tilde{\mathrm{f}}_{0}(\mathbf{q}, \mathbf{k}, t)=\lim _{\sigma \rightarrow 0} \tilde{\mathbf{f}}_{\psi}(\mathbf{q}, \mathbf{k}, t)
$$

where

$$
\tilde{\mathrm{f}}_{\psi}(\mathbf{q}, \mathbf{k}, t) \equiv \psi^{*}\left(\mathbf{q}-\frac{\sigma \mathbf{k}}{2}, t\right) \psi\left(\mathbf{q}+\frac{\sigma \mathbf{k}}{2}, t\right)
$$

and $\psi=\sqrt{\mathrm{n}} \exp (i S / \sigma)$. However, when $k \rightarrow 0$

$$
S\left(\mathbf{q} \pm \frac{\sigma_{0}}{2} \mathbf{k}, t\right)=S(\mathbf{q}, t) \pm \frac{\sigma_{0}}{2} \mathbf{k} \cdot \partial_{\mathbf{q}} S(\mathbf{q}, t)+\frac{\sigma_{0}^{2}}{8}\left(\mathbf{k} \cdot \partial_{\mathbf{q}}\right)^{2} S(\mathbf{q}, t) \pm \ldots
$$

and if the terms containing $\left(\sigma_{0} k\right)^{m}, m \geq 3$ are neglected, then

$$
\mathbf{k} \cdot \partial_{\mathbf{q}} S(\mathbf{q}, t)=\frac{1}{\sigma_{0}}\left[S\left(\mathbf{q}+\frac{\sigma_{0}}{2} \mathbf{k}, t\right)-S\left(\mathbf{q}-\frac{\sigma_{0}}{2} \mathbf{k}, t\right)\right]
$$

for any dimensional constant $\sigma_{0}$. Thus, within a suitable domain of $\mathbf{k}$, we may also consider in (67) $\sigma$ as a finite constant, (e.g. $\sigma=\hbar)$, such that $\mathrm{f}_{\psi}$ defined by (63),

$$
\mathrm{f}_{\psi}(\mathbf{q}, \mathbf{p}, t)=\frac{1}{(2 \pi)^{3}} \int d^{3} k \mathrm{e}^{-\mathrm{i} \mathbf{k} \cdot \mathbf{p}} \tilde{\mathrm{f}}_{\psi}(\mathbf{q}, \mathbf{k}, t)
$$

is the Wigner transform [11] of $\psi(\mathbf{q}, t)$. In this case, the normalization condition (61) takes the form

$$
\int \mathrm{d}^{3} q \mathrm{~d}^{3} p \quad \mathbf{f}_{\psi}(\mathbf{q}, \mathbf{p}, t)=\int d^{3} q \quad|\psi(\mathbf{q}, t)|^{2}=\langle\psi \mid \psi\rangle=\mathrm{N}
$$


and the phase-space overlap between two distributions $\mathrm{f}_{\psi_{1}}, \mathrm{f}_{\psi_{2}}$, (resembling the inner product (13)), is [9]

$$
<\mathrm{f}_{\psi_{1}} \mathrm{f}_{\psi_{2}}>\equiv \int \mathrm{d}^{3} q \mathrm{~d}^{3} p \quad \mathrm{f}_{\psi_{1}} \mathrm{f}_{\psi_{2}}=\frac{\left|\left\langle\psi_{1} \mid \psi_{2}\right\rangle\right|^{2}}{(2 \pi \sigma)^{3}}
$$

where

$$
\left\langle\psi_{1} \mid \psi_{2}\right\rangle \equiv \int \mathrm{d}^{3} q \quad \psi_{1}^{*}(\mathbf{q}, t) \psi_{2}(\mathbf{q}, t) .
$$

Worth noting, within this framework can be defined overlaps $<\mathrm{f}_{0_{1}} \mathrm{f}_{\mathrm{O}_{2}}>$ between "action distributions", or mixed overlaps $\left\langle\mathrm{f}_{\psi} \mathrm{f}_{0}\right\rangle$, while $\mathrm{f}$ can be a sum $\mathrm{f}=\mathrm{f}_{0}+\mathrm{f}_{\psi}$.

Usually, derivatives are replaced by finite differences such as (65) in numerical or lattice [28] calculations, as a result of discretization. Variations in the length unit (measure), with respect to a connection form provided by the electromagnetic potentials, have also been introduced by $\mathrm{H}$. Weyl $[29,5]$. Presuming that canonical and mechanical momentum coincide, let $\left\{\mathbf{a}_{1}, \mathbf{a}_{2}, \mathbf{a}_{3}\right\}$ be the (covariant) fundamental vectors of a Bravais lattice $\mathcal{B}_{P}$ in the momentum space, $\left\{\mathbf{a}^{1}, \mathbf{a}^{2}, \mathbf{a}^{3}\right\}$ the (contravariant) fundamental vectors of the reciprocal lattice $\mathcal{B}_{P}^{*}$,

$$
\mathbf{a}^{l}=2 \pi \epsilon_{l m n} \frac{\mathbf{a}_{m} \times \mathbf{a}_{n}}{\Omega_{P}}, \quad \Omega_{P}=\mathbf{a}_{1} \cdot\left(\mathbf{a}_{2} \times \mathbf{a}_{3}\right)
$$

and $\left\{\mathbf{c}^{i}=\hbar \mathbf{a}^{i}, i=1,2,3\right\}$ the fundamental vectors of a Bravais lattice $\mathcal{B}_{Q}$ in the coordinate space. Thus, if $\tilde{\mathbf{f}}(\mathbf{q}, \mathbf{k}, t)$ has significant values only when $\mathbf{k}$ relates two nodes of $\mathcal{B}_{P}^{*}$ and $\sigma \mathbf{k}$ in (67) relates two nodes of $\mathcal{B}_{Q}$, then $\sigma=\hbar$.

These considerations indicate that a natural relationship between the classical distribution function $\mathrm{f}_{0}$ and the quantum WKB wave function arises by phase-space discretization in elementary cells of volume $\Omega_{P} \Omega_{Q}=\mathrm{h}^{3}$, $\Omega_{Q}=\mathbf{c}^{1} \cdot\left(\mathbf{c}^{2} \times \mathbf{c}^{3}\right)$, using for the canonically conjugate variables a direct lattice and its reciprocal. It can also be shown [8] that the Wigner function $\mathbf{f}_{\psi}$ is an exact solution of (62) with $\psi$ an exact solution of the Schrödinger equation, only if the potential $V$ is a polynomial of degree at most 2 .

\section{$5 \quad$ Summary and Conclusions}

The integrality conditions of the old quantum mechanics, as well as the correspondence between observables and operators, sought in algebraic quantization, may receive a geometrical interpretation in the theory of complex 
line bundles with connection and Hermitian structure. The basic elements of this "prequantization" theory have been presented in Section 2. However, the meaning of the complex line over a (physical or reduced) phase-space $(M, \omega)$ is not obvious. The distribution function $\mathrm{f} \geq 0$ used in classical statistical mechanics is integrable over $M$, but real, while the quantum wave functions $\psi$ are complex, but $\bar{\psi} \psi$ is integrable only over the configuration space. To retrieve this property, the prequantum Hilbert space can be reduced, as indicated in Section 3, by selecting a polarization.

For the real polarization $\Lambda_{S}$ generated by the solution $S$ of the HamiltonJacobi equation, the polarized sections take the form of the quantum wave functions in the WKB approximation. However, similar functions appear in the structure of the exact solution $f_{0}$ for the classical one-particle Liouville equation, which becomes the Wigner transform $\mathrm{f}_{\psi}$ of $\psi$ if the configuration space is discretized. Although $\mathrm{f}_{\psi}$ is not positive definite, it is integrable over $M$, and the phase-space overlap between two such functions $\mathrm{f}_{\psi_{1}}, \mathrm{f}_{\psi_{2}}$ is proportional to $\left|\left\langle\psi_{1} \mid \psi_{2}\right\rangle\right|^{2}$.

By discretization, $\mathrm{f}$ aquires a nonlocal character, as $\mathrm{f}_{\psi}(\mathbf{q}, \mathbf{p})$ depends not only on $|\psi(\mathbf{q})|^{2}$, but also on $\bar{\phi}_{\mathbf{p}}\left(\mathbf{q}_{-}\right) \phi_{\mathbf{p}}\left(\mathbf{q}_{+}\right)$with $\phi_{\mathbf{p}}(\mathbf{q})=\exp (-\mathbf{i q} \cdot \mathbf{p} / \hbar) \psi(\mathbf{q})$ and $\mathbf{q}_{ \pm}=\mathbf{q} \pm \hbar \mathbf{k} / 2$ covering a whole domain of "quantum coherence", containing q. The relationship between a possible lattice structure of the phase-space and the statistical interpretation of $f_{\psi}$ remains a subject worth of further consideration.

\section{Appendix}

Definition 1. $M$ is a complex manifold if it possesses an atlas $\left\{\left(U_{i}, \varphi_{i}\right), i \in\right.$ $I\}$ where $U$ are open sets covering $M, \varphi_{i}: U_{i} \mapsto \mathcal{O}_{i} \subset \mathbb{C}^{n}$ is a diffeomorphism, and the transition functions $c_{i j}=\varphi_{j} \circ \varphi_{i}^{-1}$ are holomorphic. If $p \in U_{i} \cap U_{j}$ then $T_{p} \varphi_{i}: T_{p} M \mapsto \mathbb{C}^{n}, T_{p} \varphi_{j}: T_{p} M \mapsto \mathbb{C}^{n}$, and $T_{p} \varphi_{i} \circ\left(T_{p} \varphi_{j}\right)^{-1} \in \mathrm{GL}(n, \mathbb{C})$.

Definition 2. Let $(M, \omega)$ be a complex symplectic manifold. Then $M$ is called a Kähler manifold if for any $p \in M$ the complex structure $J_{p} \in$ $\operatorname{Sp}\left(T_{p} M\right)$ and $\omega_{p}$ define a Kähler structure on $T_{p} M, \omega_{p}\left(J_{p} x, J_{p} y\right)=\omega_{p}(x, y)$. $M$ is a positive Kähler manifold if $(x, y)_{p} \equiv \omega_{p}\left(x, J_{p} y\right)$ is positive definite. 


\section{References}

[1] Abraham R. and Marsden J., Foundations of Mechanics, Benjamin, New York, 1978.

[2] De Biévre S. and El Gradechi A., Quantum Mechanics and Coherent States on the Anti-de Sitter Spacetime and their Poincaré Contractions, Ann. Inst. Henri Poincaré 57 (1992) 403-428.

[3] Bohr N., On the Series Spectrum of Hydrogen and the Structure of the Atom, Philosophical Magazine 29 (1915) 332-335.

[4] Briggs J. and Rost J., Time Dependence in Quantum Mechanics, Eur. Phys. J. D 10 (2000) 311-318.

[5] Cartan E., Le Parallelisme Absolu et la Théorie Unitaire du Champ, Hermann et cie, Paris, 1932.

[6] Grigorescu M., Physical Framework of Quantization Problem, Rom. Rep. Phys. 45 (1993) 645-650.

[7] Grigorescu M., Constrained Evolution in Hilbert Space and Requantization, Rom. J. Phys. 38 (1993) 859-871.

[8] Grigorescu M., Classical Probability Waves, Physica A 387 (2008) 64976504 .

[9] Grigorescu M., Relativistic Probability Waves, Rev. Roumaine Math. Pures Appl. 55 (2010) 131-146.

[10] Guillemin V. and Sternberg S., Symplectic Techniques in Physics, Cambridge University Press, 1984.

[11] Gutzwiller M., Chaos in Classical and Quantum Mechanics, Springer, New York, 1990, p. 241.

[12] Hirzebruch F., Neue Topologishe Methoden in der Algebraischen Geometrie, Springer, 1956.

[13] Keller J., Corrected Bohr-Sommerfeld Quantum Conditions for Nonseparable Systems, Ann. Phys. 4 (1958) 180-188.

[14] Kostant B., Quantization and Unitary Representations, in Lecture Notes in Mathematics 170, Springer, New York, 1970.

[15] Mackey G., Induced Representations of Groups and Quantum Mechanics, W. A. Benjamin Inc., 1968.

[16] Mladenov I. and Tsanov V., Geometric Quantization of the Multidimensional Kepler Problem, J. Geometry and Physics 8 (1985) 17-24.

[17] Mladenov I. and Tsanov V., Geometric Quantisation of the MIC-Kepler Problem, J. Phys. A: Math. Gen. 20 (1987) 5865-5871. 
[18] Mladenov I., Reductions and Quantization, Int. J. Theor. Phys. 28 (1989) $1255-1267$.

[19] Mladenov I., Quantization on Curved Manifolds, In: Geometry, Integrability and Quantization, I. Mladenov and G. Naber (Eds), Coral Press, Sofia, 2001, pp. 64-104.

[20] Mladenov I., Raychev O. and Roussev R., Geometric Quantization of a Restricted Symplectic Model of Collective Nuclear Motion, Bulg. J. Phys. 13 (1986) 13-26.

[21] Planck M., Zur Theorie des Gesetzes der Energieverteilung im Normalspectrum, Verhandl. Deut. Physik. Ges. 2 (1900) 237-245.

[22] Rosensteel G. and Ihrig E., Geometric Quantization of the $\mathrm{CM}(3)$ Model, Ann. Phys. 121 (1979) 113-130.

[23] Rowe D., Ryman A. and Rosensteel G., Many-Body Quantum Mechanics as a Symplectic Dynamical System, Phys. Rev. A 22 (1980) 23622373.

[24] Śniatycki J., Geometric Quantization and Quantum Mechanics, Springer, New York, 1980, p. 198.

[25] Sommerfeld A., Zur Quantentheorie der Spektrallinien, Annalen der Physik 356 (1916) 1-94, 125-167.

[26] Sommerfeld A., Thermodynamik und Statistik, Akademische Verlagsgesellschaft, Leipzig, 1962.

[27] Voros A., Wentzel-Kramers-Brillouin Method in Bargmann Representation, Phys. Rev. A 40 (1989) 6814-6825.

[28] Wen Y., Moran Sets and Schrödinger Operators, In: Fractal Geometry and Stochastics III, C. Brandt, U. Mosco and M. Zähle (Eds), Birkhäuser, Basel-Boston-Berlin, 2004, pp. 43-56.

[29] Weyl H., Gravitation und Elektrizität, Sitz. Kön. Preuss. Akad. Wiss. Berlin (1918) 465-480.

[30] Woodhouse N., Geometric Quantization, Oxford University Press, 1980. 